\title{
O “Ensaio Para As Cônicas” de Blaise Pascal
}

\author{
João F. N. B. Cortese \\ Universidade de São Paulo - USP - Brasil \\ $\&$ \\ Laboratoire SPHERE - França \\ (aceito para publicação em agosto de 2021)
}

\begin{abstract}
Resumo
Apresentamos aqui uma tradução do Essai pour les coniques, publicado em francês por Blaise Pascal em 1640. A tradução é acompanhada de notas explicativas aos termos, assim como de breves formulações modernas dos resultados matemáticos. A introdução contextualiza o Essai pour les coniques em seu período histórico, relacionando-o aos trabalhos de Pascal e de Girard Desargues sobre as cônicas a partir de uma abordagem "perspectiva", ou, como seria mais tarde denominada, geometria projetiva. Abordamos brevemente o conteúdo matemático do tratado, em particular no que diz respeito ao dito Teorema de Pascal ou Teorema do Hexágono e à unificação de casos geométricos envolvendo o infinito e o finito.
\end{abstract}

Palavras-chave: História da Matemática, Matemática do século XVII, Blaise Pascal, Geometria Projetiva, Curvas Cônicas.

\section{[Blaise Pascal’s Essai Pour les Coniques - A Portuguese Translation]}

\begin{abstract}
We present a portuguese translation of the Essai pour les coniques, published in French by Blaise Pascal in 1640. The translation is accompanied by explanatory notes on the terms, as well as brief modern formulations of the mathematical results. The introduction contextualizes the Essai pour les coniques in its historical period, relating it to the works of Pascal and Girard Desargues on conics from a "perspective" approach, or, as it would later be called, projective geometry. We briefly discuss the mathematical content of the treatise,
\end{abstract}


in particular with regard to the so-called Pascal Theorem or Hexagon Theorem, and the unification of geometric cases involving the infinite and the finite.

Keywords: History of Mathematics, 17th century Mathematics, Blaise Pascal, Projective Geometry, Conic Curves.

\section{Introdução}

\subsection{O Ensaio para as cônicas: contexto histórico}

O Essai pour les coniques (1640) foi a primeira publicação de Blaise Pascal (1623-1662), quando este tinha apenas dezesseis anos. O propósito de Pascal com essa publicação parecia ser o de chamar a atenção da comunidade científica para as suas descobertas, inclusive indicando a data de sua realização, assim como o de anunciar a elaboração de um trabalho de maior fôlego (os Éléments coniques complets referidos no texto), e, a crer na conclusão modesta do texto, o de receber reações dos leitores sobre o conteúdo apresentado aí (MESNARD, em OC, II, p. 220) ${ }^{1}$. Trata-se, portanto, de um "ensaio”, no sentido próprio do termo: mais do que uma obra concluída, Pascal apresenta um programa de trabalho elaborado a partir do que Girard Desargues (1591-1661) já encontrara sobre a abordagem perspectiva das cônicas. Justifica-se aí que se trate de um ensaio "para” as cônicas: a saber, para um tratado sobre as cônicas que Pascal anunciava que viria a apresentar.

Infelizmente, deste trabalho mais amplo de Pascal sobre as cônicas não nos restam hoje senão fragmentos, em particular o primeiro trecho do tratado, intitulado Geração das seções cônicas, que foi copiado por Leibniz, assim como notas deste sobre as partes que constituíam o texto completo ${ }^{2}$. O fato de o tratado de Pascal sobre as cônicas ter sido perdido quase por inteiro nos leva a valorizar ainda mais o Ensaio para as cônicas, apesar de se tratar de uma obra curta.

Todo o Ensaio para as cônicas foi editado em uma única página, de $35 \mathrm{~cm}$ por $43 \mathrm{~cm}$, (MESNARD, em OC, II, p. 220), como um tipo de panfleto. Suas cinquenta cópias não foram vendidas, mas distribuídas entre estudiosos da época.

\footnotetext{
${ }^{1}$ Citamos por OC a edição de Jean Mesnard das obras completas de Pascal (PASCAL, 1964-1992).

${ }^{2} \mathrm{O}$ tratado de Pascal sobre as cônicas, que foi referido como Traité des coniques, foi anunciado pelo autor como Conicorum opus completum em uma carta dedicatória à academia parisiense (Celleberrimae Matheseos Academiae Parisiensi), de 1654 (OC, II, p. 1033). Escrito em latim, resta dele apenas o trecho inicial, copiado por Leibniz, intitulado Generatio conisectionum, tangentium et secantium; seu projectio peripheriae, tangentium, et secantium circuli in quibuscumque oculi, plani ac tabellae positionibus (OC, II, p. 1108), ou seja, Geração das seções cônicas, tangentes e secantes; ou projeção da circunferência, tangentes e secantes do círculo para cada posição do olho e do plano do quadro. Restam também notas sobre esse tratado realizadas por Leibniz, que teve o manuscrito em mãos e recomendou sua publicação, a qual infelizmente nunca ocorreu (ver OC, II, pp. 1102-1131; ver também CORTESE, 2016). Para detalhes históricos sobre as obras de Pascal sobre as cônicas, ver J. Mesnard em OC (II, pp. 220-228, 1021-1031, 1102-1108). Alguns autores se propuseram a reconstruir a ambição completa do tratado de Pascal sobre as cônicas - ver TATON, 1962 e DEL CENTINA, 2020.
} 


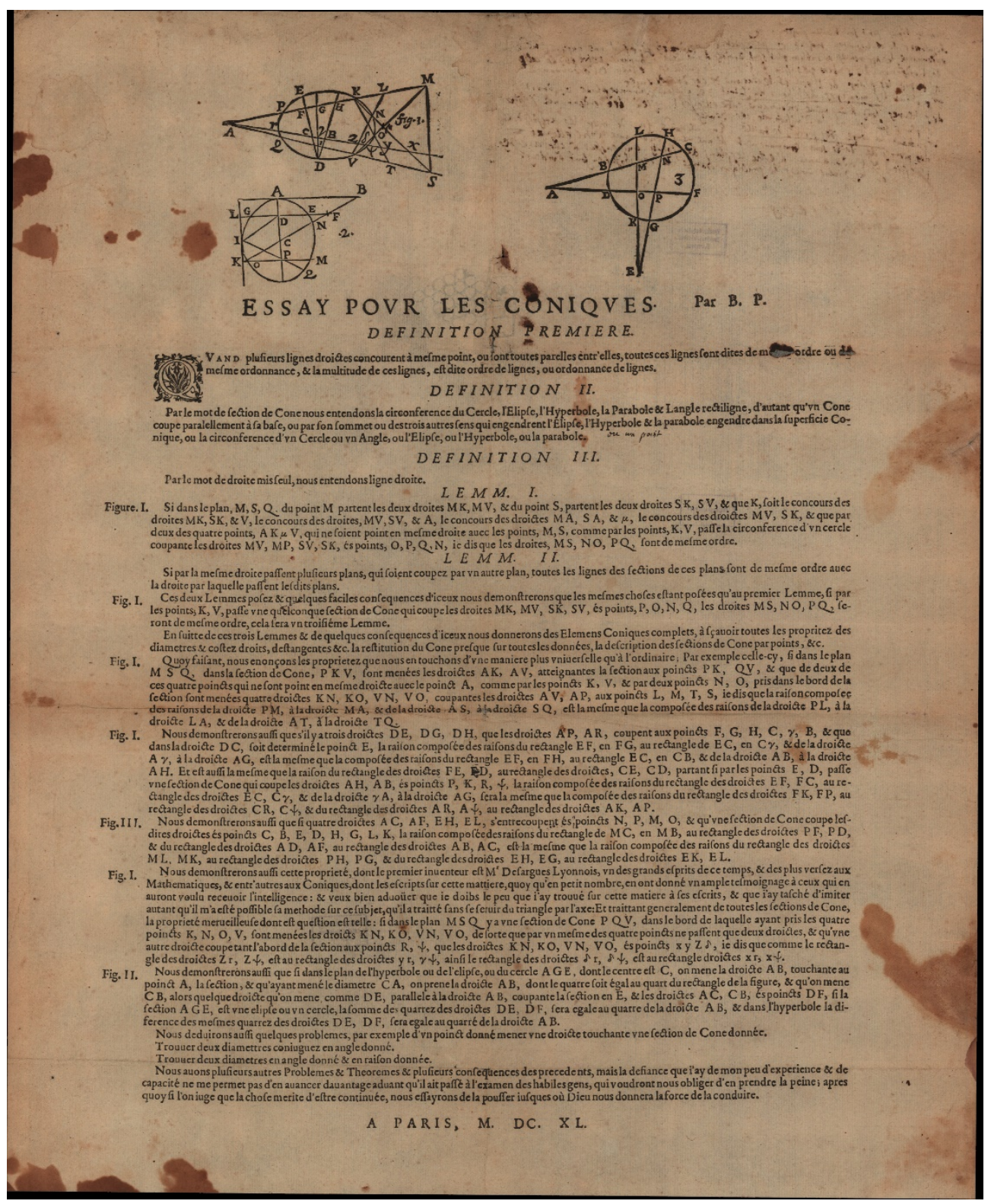

(Essay pour les coniques, LH XXXV, XV, I, Bl. 10v. Imagem em alta resolução gentilmente disponibilizada pela G.W. Leibniz Bibliothek - Niedersächsische Landesbibliothek, Hannover) 
Trata-se, entretanto, de um panfleto que teve uma grande repercussão, pois ele apresentava um caso particular do que seria depois chamado de Teorema de Pascal, ou Teorema do Hexágono: o alinhamento dos pontos de intersecção de três pares de lados opostos de um hexágono inscrito em uma cônica. Além disso, ele apresentava uma abordagem para o estudo das cônicas que pode ser dita "projetiva”. Antes de tratarmos de como este teorema é apresentado, vejamos brevemente quais são os conteúdos matemáticos do Ensaio para as cônicas, assim como qual sua relação com a “geometria projetiva”.

\subsection{O conteúdo matemático do Ensaio para as cônicas}

O Ensaio para as cônicas apresenta três definições, três lemas e cinco teoremas, todos apresentados sem demonstrações, além de três problemas de construção, brevemente enunciados ${ }^{3}$.

A primeira definição, de retas de mesma “ordem” (ordre), ou de mesma "ordenança” (ordonnance), permite a Pascal tratar simultaneamente de retas concorrentes e de retas paralelas. A segunda definição, de "seção cônica”, revela como Pascal concebe de modo unificado as seções cônicas (círculo, elipse, hipérbole, parábola e ângulo retilíneo) ${ }^{4}$. A terceira definição traz uma precisão de vocabulário para as "retas”.

O primeiro lema apresenta uma versão do Teorema de Pascal para o caso do círculo, e o terceiro lema uma versão do teorema para uma cônica qualquer. Na verdade, trata-se de um resultado equivalente, pois aqui não é questão de hexágono - ver abaixo, seção 1.4. O segundo lema apresenta um resultado simples de geometria espacial.

Pascal anuncia então deduzir diversas consequências desses lemas e de suas consequências, o que constituiria um tratado completo sobre as cônicas. A título de exemplo desta abordagem geral, ele apresenta na sequência do texto cinco teoremas.

"O primeiro desses teoremas afirma a igualdade das razões anarmônicas $^{5}$ dos feixes de retas que unem quaisquer dois pontos de uma

\footnotetext{
${ }^{3}$ Com relação ao conteúdo matemático do texto, nos baseamos fundamentalmente em TATON, 1955; HOUZEL, 2013 e DEL CENTINA, 2020.

${ }^{4}$ Na Generatio conisectionum, Pascal indicará a existência de seis seções cônicas: a elipse (chamada por ele de antobola), a parábola, a hipérbole, e os casos ditos "degenerados" que são o ponto (o vértice do cone), a reta (que chamamos hoje de geratriz) e o ângulo retilíneo, oriundo de uma seção que passa pelo vértice e que divide o cone em duas partes iguais (OC, II, pp. 1110-1111; cf. CORTESE, 2016, pp. 147-148). A circunferência do círculo, que era considerada como uma das seções cônicas no Ensaio para as cônicas, não o é mais na Generatio conisectionum. É claro que a circunferência do círculo pode ser entendida como um caso da elipse. Mas essa mudança de classificação por Pascal não significa que a circunferência era considerada como de pouca importância neste último texto: ao contrário, as generalizações da geometria projetiva são feitas aí a partir da consideração de cada uma das seções cônicas como uma "imagem da circunferência do círculo" (OC, II, p. 1113).

${ }^{5}$ A razão anarmônica, também chamada de razão dupla ou razão cruzada (em inglês, cross-ratio) pode ser assim definida: dados quatro pontos colineares (A, B, C, D), sua razão anarmônica é definida por
}

$A B \times C D$

$\overline{A D \times C B}$

(cf. FIELD e GREY, 1987, p. 52). Pode-se notar tal razão anarmônica como (ABCD). Tal razão é invariante sob transformações projetivas.

RBHM, Vol. 21, n 42, pp. 180-205, 2021 
cônica com 4 pontos dados nesta mesma curva. O segundo teorema inclui várias proposições: o teorema dito de Pappus sobre a igualdade das razões anarmônicas dos grupos de pontos determinados sobre duas secantes por um feixe de 4 retas, o teorema clássico dito de Menelau (ou Ptolomeu) sobre as razões dos segmentos determinados por uma secante nos lados de um triângulo e, finalmente, um caso particular do teorema dito de Carnot sobre as razões dos segmentos determinados por uma cônica nos 3 lados de um triângulo. O terceiro teorema, uma extensão do anterior, e outro caso particular do teorema dito de Carnot, expressa a relação que satisfazem os segmentos determinados por uma seção cônica nos lados de um quadrilátero. A quarta proposição é o famoso teorema de Desargues sobre a involução determinada em uma secante qualquer por uma seção cônica e os lados de um quadrilátero inscrito. Finalmente, o Teorema $V$, mal interpretado pela maioria dos comentadores, é equivalente às equações clássicas das cônicas centrais, relativas a dois diâmetros conjugados." ${ }^{6}$ (TATON, 1955, p. 7)

Finalmente, Pascal enuncia ao final do texto certos problemas de construção, que podem ser tratados através de seu método.

\subsection{A "geometria projetiva" de Pascal}

O Ensaio para as cônicas foi escrito sob a influência direta de Girard Desargues, e foi publicado em 1640, ou seja, apenas um ano após o célebre Brouillon project d'une Atteinte aux evenemens des rencontres du cone avec un plan (1639) deste ${ }^{7}$. Neste tratado, de linguagem difícil e pouco lido mesmo pelos matemáticos da época, Desargues apresentava uma abordagem "perspectiva" das cônicas que foi fundamental para Pascal.

Além da influência direta de Desargues, Pascal provavelmente conheceu dois trabalhos clássicos sobre as cônicas: as Cônicas de Apolônio de Perga (que Pascal cita na carta de 1654 à "Academia”) e a Coleção de Pappus de Alexandria (cf. TATON, 1962, p. 207). Ambos haviam sido editados por Federico Commandino em Bolonha, respectivamente em 1566 e 1588, e eram bem conhecidos pelos matemáticos do século XVII (cf. Mesnard em OC, II, p. 221). De fato, alguns trabalhos sobre as cônicas existiam nesse período - o matemático francês Claude Mydorge, amigo de Étienne Pascal, pai de Blaise, publicara um trabalho sobre as cônicas em 1631, com o qual Pascal era provavelmente familiar (TATON, 1962, p. 207). Nenhum desses trabalhos, entretanto, trazia uma abordagem "perspectiva" das cônicas tal como feita por Desargues ${ }^{8}$.

\footnotetext{
${ }^{6}$ No caso das cônicas centrais, os diâmetros passam pelo centro da cônica. Pode-se definir dois diâmetros como “conjugados” se cada corda paralela a um diâmetro é bisseccionada pelo outro diâmetro (cf. por ex. NABONNAND, 2012).

${ }^{7}$ A edição das obras de Desargues foi feita por R. TATON, 1951. É preciso hoje consultar a edição de 1981 (TATON, 1981, reimpressa em 1988), na qual são corrigidos certos erros (do próprio Desargues). Uma tradução das obras geométricas para o inglês foi feita por FIELD e GRAY, 1987.
} 
Mas em que consistia tal abordagem? Desde o século XIV, a abordagem da perspectiva linear na pintura italiana permitia a representação de retas paralelas que se encontravam em um ponto de fuga. Desargues trabalhou sobre uma nova geometria, a qual, usando um método "perspectivo", tratava as cônicas como projeções umas das outras. Os resultados encontrados por ele seriam mais tarde incorporados ao campo que veio a se chamar "geometria projetiva" .

Essa técnica projetiva (ou "perspectiva") aparece nas obras de Desargues e de Pascal no contexto do estudo das cônicas, permitindo uma generalização de resultados para estas: uma cônica sendo projeção de outra, um mesmo resultado pode ser demonstrado simultaneamente para ambas (desde que se trate de uma propriedade invariante sob projeção). De fato, considerar as seções cônicas como projeções do círculo permite a generalização de vários teoremas. Como dissemos, geômetras antigos como Apolônio e Pappus já haviam estudado as cônicas, revelando várias de suas propriedades, mas a geometria projetiva de Desargues e Pascal foi além, tratando os teoremas correspondentes de uma maneira unificada. Fundamental para este projeto de unificação foi a admissão de elementos a distância infinita.

Assim como Desargues, Pascal proporá na Generatio conisectionum que quaisquer duas retas se encontram, seja a distância finita, seja a uma distância infinita (neste último caso, as retas sendo paralelas; OC, II, p. 1111). Quanto ao Ensaio para as cônicas, Pascal também trata o caso infinito e o finito conjuntamente, mas por meio de outro terminologia: Desargues chamara às retas que se encontram em um ponto (a distância finita ou infinita) como sendo de mesma "ordenança”, vocabulário que Pascal retoma, apresentando o termo como sinônimo de "ordem" (ver a primeira definição do texto traduzido abaixo). Podemos ver então como a abordagem projetiva, e seu papel fundamental na unificação de casos geométricos, já aparece esboçada no Ensaio para as cônicas.

\subsection{0 “Teorema de Pascal”}

Tal definição será de particular importância na formulação do célebre Teorema do Hexágono, dito Teorema de Pascal. Consideremos, no que diz respeito a ele, o primeiro lema do Ensaio para as cônicas - em relação à figura A (seção 2.2), o Pascal formula assim (ver tradução à seção 3):

"Se, no plano MSQ, do ponto $M$ partem as duas retas $M K, M V$, e do ponto $S$ partem as duas retas $S K, S V$, e sendo K a concorrência das retas $M K, S K$, e $V$ a concorrência das retas $M V$, SV, e A a concorrência das

\footnotetext{
${ }^{8}$ A importância das curvas cônicas nas ciências do século XVII não deve ser negligenciada: Kepler identifica órbitas elípticas de planetas na sua Astronomia Nova (1609) e hipérboles para a medida das refrações no Ad Vitellionem paralipomena (1604); Galileu estuda em seus Discorsi (1638) a trajetória parabólica dos projéteis; Descartes usa todas essas cônicas na Geometria (1637) para resolver o problema de Pappus, e avalia na Dióptrica se a forma ideal das lentes é parabólica ou hiperbólica (cf. DHOMBRES 1994). As cônicas são fundamentais tanto na mecânica quanto na ótica durante esse período, e Mersenne menciona na sua Harmonia universal (1636) até mesmo aplicações acústicas para esse tipo de curvas. No entanto, deve-se lembrar que as cônicas podem ser estudadas sem a geometria projetiva: esta é apenas uma das abordagens possíveis ao estudo das cônicas.

${ }^{9}$ A este respeito, ver, por exemplo, BKOUCHE, 1991 e ANDERSEN, 2007.
} 
retas $M K, S V$, e $\mu$ a concorrência das retas $M V, S K$, e que por dois dos quatro pontos $A, K, \mu, V$, que não estejam em uma mesma reta com os pontos $M, S$, como pelos pontos $K, V^{10}$, passe a circunferência de um círculo, que corta as retas $M V, M K, S V$, $S K$, nos pontos $O, P, Q, N$; digo que as retas $M S, N O, P Q$, são de mesma ordem."

Podemos entender daqui que, para o hexágono $P K N O V Q$ inscrito na circunferência, se seus lados opostos $P K, O V$ se encontram em $M$, e se seus lados opostos $K N, V Q$ se encontram em $S$, então os lados opostos $N O, Q P$ se encontram em um ponto sobre a reta MS (cf. HOUZEL, 2013, p. 91). Ou seja, as intersecções encontradas por cada um dos três pares de lados opostos são colineares ${ }^{11}$.

O curioso a ser notado é que, nesta formulação original do lema I, diferentemente do que é o caso no "Teorema do Hexágono", Pascal não fala nem de "hexágono" nem de "hexagrama”. Sua formulação passa antes pela noção de "ordem'”: são de mesma ordem as retas que concorrem em um mesmo ponto, tais como, neste caso, $P Q, N O$ e $M S$. Tal noção de ordem compreende inclusive o caso das retas paralelas, no qual também se diz que essas retas se encontram - a uma distância infinita. Para o caso do teorema em questão, os lados opostos se tornam paralelos quando se considera um hexágono regular.

Quanto ao terceiro lema do Ensaio para as cônicas, ele apresenta uma extensão do lema I, onde ao invés do círculo considera-se uma cônica qualquer. Como indica Houzel (2013), ele pode ser derivado diretamente do lema I, caso se considere a cônica como projeção do círculo e notando que a propriedade em questão é invariante sob projeção. Aqui aparece então um resultado equivalente ao Teorema de Pascal para qualquer cônica - mas tampouco é feita menção a um hexágono.

Cabe também considerar o célebre termo de "hexagrama místico", que Leibniz relata que estaria no tratado das cônicas de Pascal, ainda que não tenhamos nenhuma evidência direta de que Pascal tenha usado tal terminologia ${ }^{12}$. Entretanto, como apontado pelo próprio Leibniz, haveria uma diferença entre o "hexagrama místico" e o "hexagrama cônico"13. Para Debuiche (2016, p. 5, nota), o teorema do hexagrama místico consistiria em que, seis pontos sendo dados, se três retas determinadas pelos pontos opostos (pontos 1 e 4 , 2 e 5,3 e 6) são de mesma ordem (ou seja, paralelas ou concorrentes em um mesmo ponto), então os seis pontos estão sobre uma cônica. Tal teorema seria portanto relacionado ao

\footnotetext{
${ }^{10}$ Hara (1981, pp. 13-14) declarou que se os pontos escolhidos forem $A$ e $\mu$, ao invés de $K e V$, o teorema é parcialmente falso. Houzel (2013, p. 91), entretanto, indica que, no caso em que $A$ e $\mu$ forem os pontos escolhidos, basta trocar os papeis de $P$ e de $O$ (ou de $Q$ e de $N$ ) para recuperar o lema de Pascal, o hexágono em consideração sendo $A P N \mu O Q A$, e os lados opostos $P N$ e $O Q$ se cruzando sobre a reta MS. Houzel conclui, portanto, que o enunciado do lema contém uma imprecisão, mas que não chega a ser um erro.

${ }^{11}$ Deve-se entender que, quando se fala de intersecção dos lados aqui, em função do caso em questão cabe considerar as retas constituídas pelo prolongamento desses lados.

${ }^{12} \mathrm{Em}$ suas notas sobre o tratado das cônicas pascaliano, Leibniz escreve sobre o hexagrammum pascalianum mysticum, que ele diz ser assim referido por Pascal (cf. OC, II, p. 1129).

${ }^{13}$ Leibniz escreve (em latim): "as definições extraídas das cônicas tratam do hexagrama místico, em primeiro lugar por ele mesmo; em seguida, é questão do hexagrama cônico” (em OC, II, p. 1120); "Hexagrama pascaliano místico, como ele mesmo o chama, e também sempre cônico” (em OC, II, p. 1129). Tradução feita a partir daquela de Mesnard para o francês.
} 
primeiro lema do Ensaio para as cônicas, mas com a diferença de que ele independe do cone ou de suas seções. É assim que, segundo Debuiche, o hexagrama místico subsumiria o hexagrama cônico, a potência heurística que lhe é atribuída enquanto propriedade fundamental da geometria justificando seu lugar no início do tratado das cônicas pascaliano, logo após a apresentação da geração das seções cônicas ${ }^{14}$. Como toda cônica é definida por um hexagrama místico ${ }^{15}$, este se torna um modo de conhecer as propriedades de todas as seções cônicas ${ }^{16}$.

Um dos aspectos interessantes do Teorema do Hexágono de Pascal é que ele é válido para um hexágono inscrito em qualquer cônica, e não necessariamente em uma circunferência. Isso ilustra a enorme potência da geometria projetiva, que oferece a possibilidade de demonstrar teoremas válidos a um só tempo para cada uma das cônicas ${ }^{17}$.

Pascal provavelmente dava a seu teorema sobre o hexágono um papel central no seu Tratado sobre as cônicas ${ }^{18}$. Mersenne escreveu a respeito de Pascal e de seu Teorema do Hexágono:

"temos aqui um jovem (...) que é um geômetra tão excelente, tendo apenas 18 anos, que ele compreendeu todas as seções cônicas e o Apolônio em uma única proposição, da qual ele deriva de tal maneira 400 corolários que nenhum depende do outro, mas todos, tanto o último como o primeiro, da referida proposição." (Carta a T. Haak, 18 de novembro de 1640; em OC, II, p. 239)

Ainda que a falta de documentos nos impeça de julgar sobre a justiça de falar sobre 400 corolários derivados por Pascal de seu Teorema do Hexágono (dado que o tratado pascaliano sobre as cônicas foi perdido), fica clara a impressão que o poder generalizador da geometria projetiva causou à época.

Tais resultados, entretanto, não foram recebidos por todos com entusiasmo. A geometria projetiva, entretanto, teve uma difícil recepção, tendo sido retomada apenas na virada do século XVIII para o século XIX. Talvez isso seja devido aos aspectos contraintuitivos que ela trazia em relação à geometria euclidiana.

Descartes, em particular, apresentou ressalvas em relação ao Ensaio para as cônicas, em uma carte do $1^{\circ}$ ou de 2 de abril de 1640 a Mersenne:

\footnotetext{
${ }^{14}$ No seu relato sobre o tratado das cônicas de Pascal, Leibniz situava o tratado De Hexagrammo como segunda parte, logo após a Generatio conisectionum (cf. OC, II, p. 1120).

15 “[...] e ele [Pascal] mostra por meio das projeções que todo Hexagrama Místico convém a uma seção cônica, e que toda seção cônica dá um Hexagrama Místico” (carta de Leibniz a Étienne Périer, Paris, 30 de agosto de 1676; em LEIBNIZ 1923- , III, 1, p. 588; citado por DEBUICHE, 2016).

${ }^{16}$ Sobre o hexagrama místico do ponto de vista combinatorial e sobre os seus supostos usos, ver também Del Centina (2020, em particular seção 7.2).

${ }^{17}$ Notemos também que se pode entender o Teorema de Pappus como um caso particular do Teorema de Pascal, como tratando-se de seis pontos inscritos em uma cônica que são as duas retas formando um ângulo.

${ }^{18}$ É o que se depreende da afirmação de Pascal à “Academia Parisiense”, das notas de Leibniz e da declaração de Mersenne citada abaixo.Ver TATON, 1955, pp. 7-8.
} 
"Recebi também o Essai touchant les Coniques, do filho do Senhor Pascal $e$, antes de ter lido a sua metade, julguei que ele [o] havia aprendido do Senhor Desargues, o que me foi confirmado imediatamente pela confissão feita por ele mesmo.” (em OC, II, pp. 238-239)

Seria então Pascal apenas um jovem que replicou resultados já existentes, reproduzindo a abordagem de Desargues, e tendo como inovação apenas o Teorema do Hexágono?

"Quanto aos próprios teoremas escolhidos como exemplos, pode-se pensar, ou que, tendo sido facilmente demonstrados no caso do círculo, foram então estendidos por projeção ao caso geral, ou que foram deduzidos do teorema do hexágono inscrito. Em sua maioria, esses teoremas, além disso, não são originais. O primeiro é uma conseqüência bastante direta do teorema de Desargues. O segundo inclui duas proposições então muito conhecidas; mas sua última parte e o teorema 3 , casos particulares do chamado teorema dito de Carnot, são talvez mais originais, embora sua aplicação ao caso particular do círculo seja muito elementar. O teorema 4, retirado diretamente do Brouillon project de Desargues, dá a Pascal a oportunidade de prestar uma homenagem vibrante a seu mestre; finalmente, o teorema 5 já é encontrado de forma semelhante em Desargues, ou mesmo em Apolônio." (TATON, 1955, p. 8)

Podemos, portanto, entender que, mais do que resultados originais, Pascal deseja apresentar resultados geométricos segundo uma nova ordem. Esta entretanto, pode não ser considerada uma mera repetição, mas algo que se mostra fecundo quanto ao seu poder generalizador: "que não se diga que eu nada disse de novo: a disposição das matérias é nova” escrevia o próprio Pascal ${ }^{19}$.

Finalmente, lembremos que Pascal, um espírito amplo, não se limitou a fazer cálculos a partir das matemáticas, mas importou diversos conceitos e reflexões destas para um pensamento sobre o ser humano. Foi dessa maneira que ele escreveu nos seus Pensamentos, de uma maneira que lembra a problemática da perspectiva e da geometria projetiva:

"Se se considera sua obra imediatamente após tê-la feito, ainda se tem muita prevenção; se muito tempo depois, não se entra mais nela. Assim os quadros vistos de muito longe e de muito perto. E só há um ponto indivisível que é o verdadeiro lugar. Os outros estão perto demais, longe demais, alto demais ou baixo demais. A perspectiva o indica na arte e na

\footnotetext{
${ }^{19}$ Pensamentos, fragmento Lafuma 696, Sellier 575. Consultamos a edição dos Pensamentos feita por D. Descotes (PASCAL 2011), realizando uma tradução baseada naquela de PASCAL, 2005. Sobre esta frase relacionada à originalidade de Pascal na história da ciências, ver DUHEM, 1905.
} 
pintura. Mas na verdade e na moral, quem o indicará?” (Pensamentos, fragmento Lafuma 21, Sellier 55)

O ponto de vista a partir do qual se pode ordenar tudo, no caso de certas curvas, é o vértice de um cone: qual será ele no caso do ser humano e do que lhe concerne? Isso seria tema para as reflexões antropológicas de Pascal, que devem ser exploradas em outro contexto.

\subsection{Sobre a tradução do texto e sua edição}

Dois exemplares do texto original restam hoje, na Bibliothèque Nationale de France (BNF) e na G. W. Leibniz Bibliothek - Niedersächsische Landesbibliothek, em Hannover, a imagem deste último sendo reproduzida acima.

Para a edição do texto, seguimos a excelente edição de Jean Mesnard, referência atual para as obras matemáticas de Pascal (PASCAL, 1964-1992). Reproduzimos o seu texto, com suas indicações de estabelecimento de texto, assim como suas eventuais correções (apresentando o original em nota). A impressão original do texto continha diversos erros tipográficos, inclusive quanto às letras que faziam referência às figuras, o que foi corrigido por TATON (1955) e em maior medida na edição de Mesnard.

Na tradução, apresentamos as notas de Mesnard com formulações modernas dos resultados das proposições, além de outras notas relativas ao conteúdo do texto. Vale notar que, quando apresentamos frações e multiplicações para expressar proporções e "razões compostas" indicadas por Pascal, trata-se de um modo anacrônico de abordar seus resultados. Se o fazemos para auxiliar o leitor contemporâneo, a própria natureza da tradução de um texto em história da matemática tem como propósito lembrar a importância de conhecer a formulação de um resultado em seu contexto original.

Quanto a possíveis demonstrações dos resultados de Pascal, damos aqui apenas breves indicações - para maiores detalhes, ver (TATON, 1955; HARA, 1981; DEL CENTINA, 2020; e, sobretudo, HOUZEL, 2013).

Na publicação original, três figuras eram apresentadas, reunindo simultaneamente elementos de diversos teoremas. Taton (1955) desenvolveu cinco figuras a partir da figura I, que apresentamos aqui (seguindo as pequenas modificações feitas por Mesnard para uniformização das letras), ao lado das três figuras originais, com o propósito de clarificar a compreensão do texto.

Na tradução e no texto original, a referência às figuras correspondentes é apresentada entre colchetes ao final de cada proposição. ${ }^{20}$

\footnotetext{
${ }^{20}$ Na edição original, as figuras, numeradas por algarismos indo-arábicos, são designadas no texto por algarismos romanos (cf. nota de Mesnard em OC, II, p. 231).
} 


\section{Bibliografia}

ANDERSEN, Kirsti. 2007. The Geometry of an Art: The History of the Mathematical Theory of Perspective from Alberti to Monge. New York: Springer.

BKOUCHE, Rudolf. 1991. La naissance du projectif. De la perspective à la géométrie projective. In: Mathématiques et philosophie, ed. R. Rashed. Paris: CNRS, 239-285.

CARNOT, Lazare. 1806. Mémoire sur la relation qui existe entre les distances respective de cinq points quelconque pris dans l'espace; suivi d'un essai sur la théorie des transversales. Paris: Curcier.

CHASLES, Michel. 1875 ( $2^{\mathrm{a}}$ ed.). Aperçu historique sur l'origine et le développement des méthodes en géométrie... Paris.

CORTESE, João F. N. 2016. Leibniz e o paradigma da perspectiva. Cadernos Espinosanos, 34, 137-162.

COSTABEL, Pierre. 1962. Traduction française de notes de Leibniz sur les « Coniques » de Pascal. Revue d'histoire des sciences et de leurs applications, t. 15, n. 3-4, 253-268.

DEBUICHE, Valérie. 2016. L'invention d'une géométrie pure au 17e siècle: Pascal et son lecteur Leibniz. Studia Leibnitiana, 48, 42-67.

DEL CENTINA, Andrea. 2020. Pascal's mystic hexagram, and a conjectural restoration of his lost treatise on conic sections. Archive for History of Exact Sciences, v. 74, n. 5, 469521.

DHOMBRES, Jean. 1994. La culture mathématique au temps de la formation de Desargues: le monde des coniques. In: Desargues en son temps, ed. J. Dhombres e J. Sakarovitch. Paris: A. Blanchard.

DUHEM, Pierre. 1905. Le principe de Pascal: Essai historique. Revue générale des sciences pures et appliquées, t. XVI, n. 13, 599-610.

FIELD, Judith V. e GRAY, Jeremy J. 1987. The geometrical work of Girard Desargues. New York, Berlim: Springer.

HARA, Kokiti. 1981. L'œuvre mathématique de Pascal. Osaka: Osaka University Press, Memoirs of the Faculty of Letters, vol. XXI.

HOUZEL, Christian. 2013. Blaise Pascal et les sections coniques. In: Les courbes: études sur l'histoire d'un concept, ed. R. Rashed e P. Crozet. Paris: Blanchard. 
LEIBNIZ, Gottfried Wilhelm.1923- . Sämtliche Schriften und Briefe. Darmstadt, Leipzig, Berlin: Akademie-Verlag.

MESNARD, Jean. 1994. Desargues et Pascal. In: Desargues en son temps, org. J. Dhombres e J. Sakarovitch. Paris: A. Blanchard.

NABONNAND, Philippe. 2012. La théorie des coniques chez Poncelet, Steiner et von Staud. Disponível em <https://hal.archives-ouvertes.fr/hal-01079478/document. Acesso em 5/08/2021>.

PASCAL, Blaise. 1640. Essay pour les coniques. Paris. Versão consultada no acervo da G.W. Leibniz Bibliothek - Niedersächsische Landesbibliothek, Hannover (documento LH XXXV, XV, I, Bl. 10v). Disponível em $<$ http://digitale-sammlungen.gwlb.de/sammlungen/sammlungsliste/werksansicht/?id=6\&tx_ dlf\%5Bid\%5D=1742\&tx_dlf\%5Bpage\%5D=20>.

PASCAL, Blaise. 1964-1992. E Euvres Complètes. Edição de Jean Mesnard. Paris : Desclée de Brouwer, volumes I (1964), II (1970), III (1991) e IV (1992). (Citado como OC).

PASCAL, Blaise. 2005. Pensamentos. Tradução de Mario Laranjeira a partir da edição Lafuma. São Paulo: Martins Fontes.

PASCAL, Blaise. 2011. Édition électronique des Pensées de Blaise Pascal. Ed. D. Descotes e G. Proust. Disponível em <http://www.penseesdepascal.fr/>.

TATON, René. 1951. L'œuvre mathématique de G. Desargues. Paris: P. U. F.

TATON, René. 1955. L'“Essay pour les coniques” de Pascal. Revue d'histoire des sciences et de leurs applications, t. 8, n. 1, 1-18.

TATON, René. 1962. L’œuvre de Pascal en géométrie projective. Revue d'histoire des sciences et de leurs applications, t. 15, n. 3-4, 197-252.

TATON, René. 1981 (2ª ed.). L'œuvre mathématique de G. Desargues. Paris: Vrin.

\section{João F. N. B. Cortese}

Departamento de Fisiologia - IB-USP - campus de São Paulo - Brasil

$\&$

Laboratoire SPHERE - CNRS \& Université de

Paris (UMR 7219) - França

E-mail: joaocortese@gmail.com 


\section{Figuras}

2.1 As três figuras originais da publicação de Pascal (reprodução a partir do proposto por Mesnard, em OC, II, pp. 228-229) ${ }^{21}$

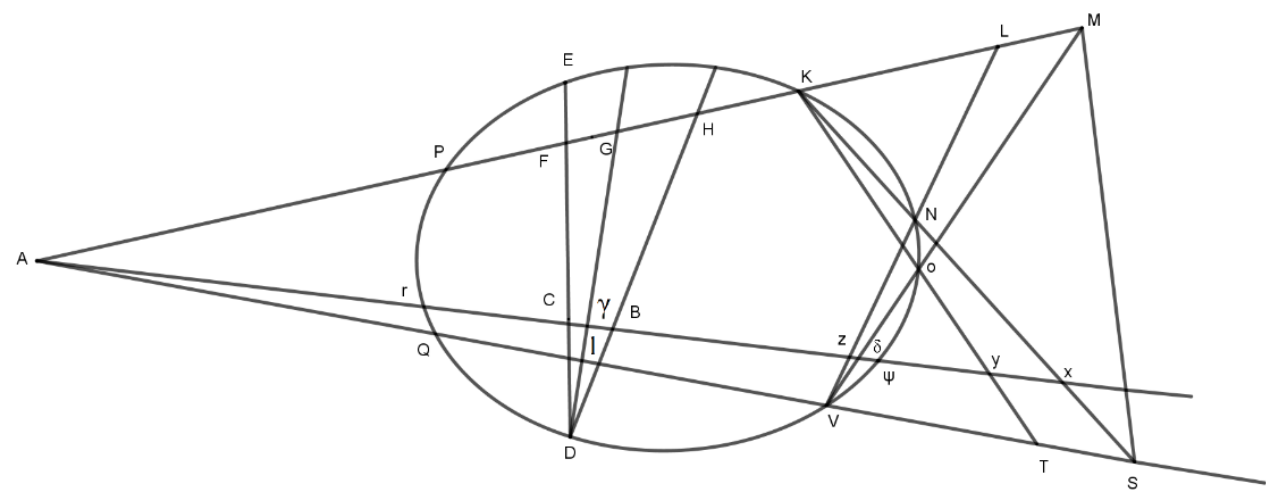

Figura I

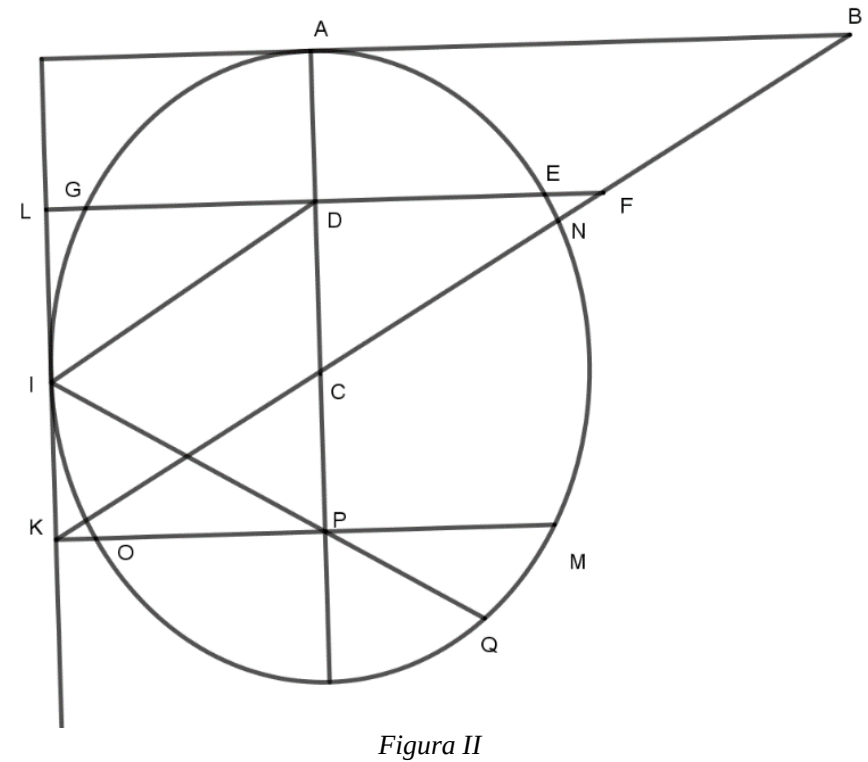

${ }^{21} \mathrm{~A}$ única alteração que fazemos é uma correção à figura de Mesnard, na qual o y é confundido com o Y. 


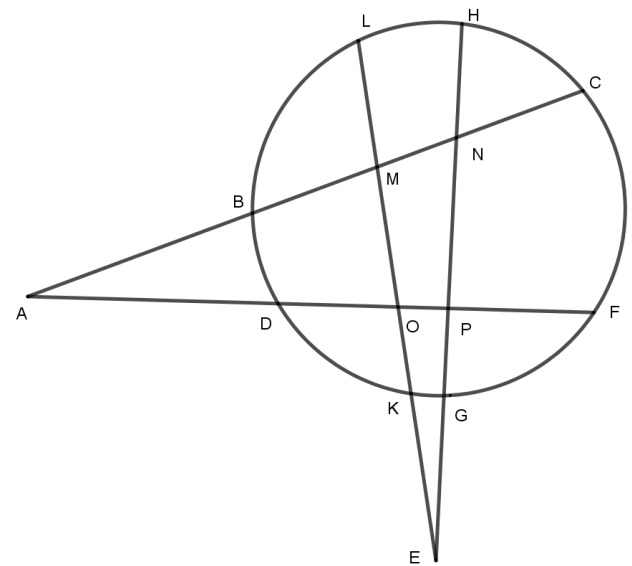

Figura III

RBHM, Vol. 21, n 42, pp. 180-205, 2021 
2.2 Figuras auxiliares (a partir da decomposição da figura I realizada por TATON, 1955; ver também OC, II, pp. 229-230)

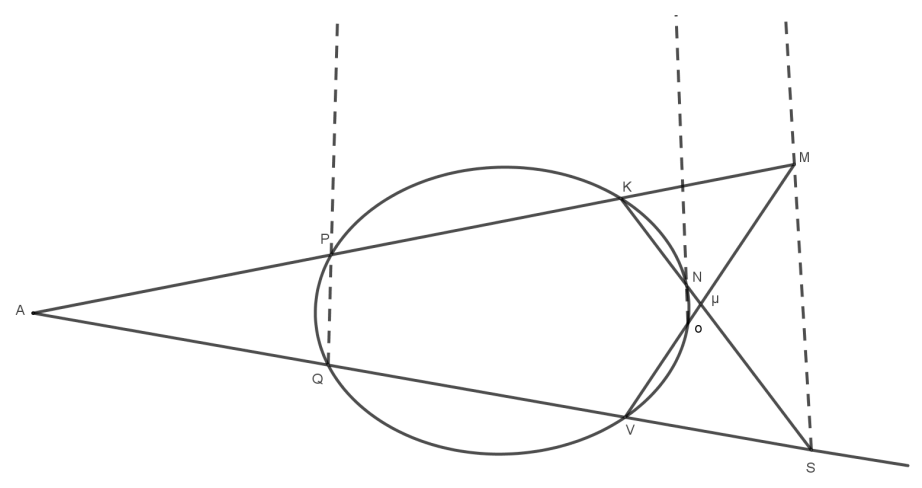

Figura A

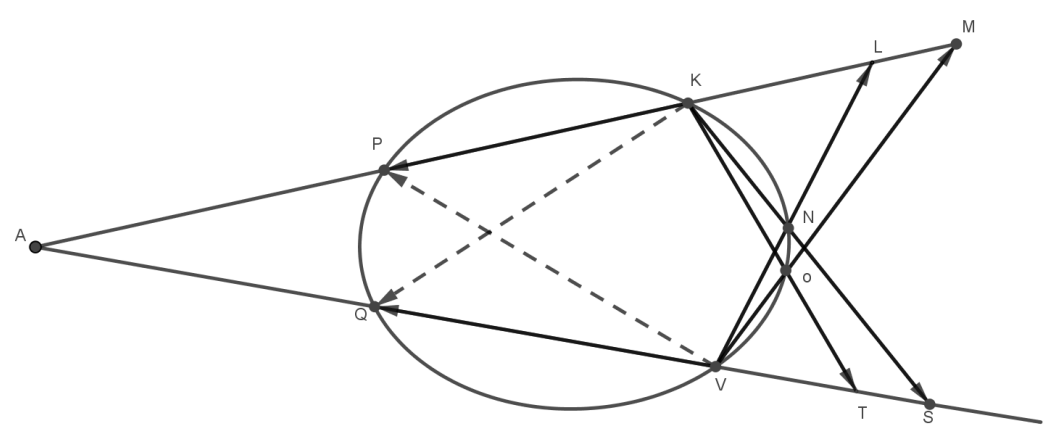




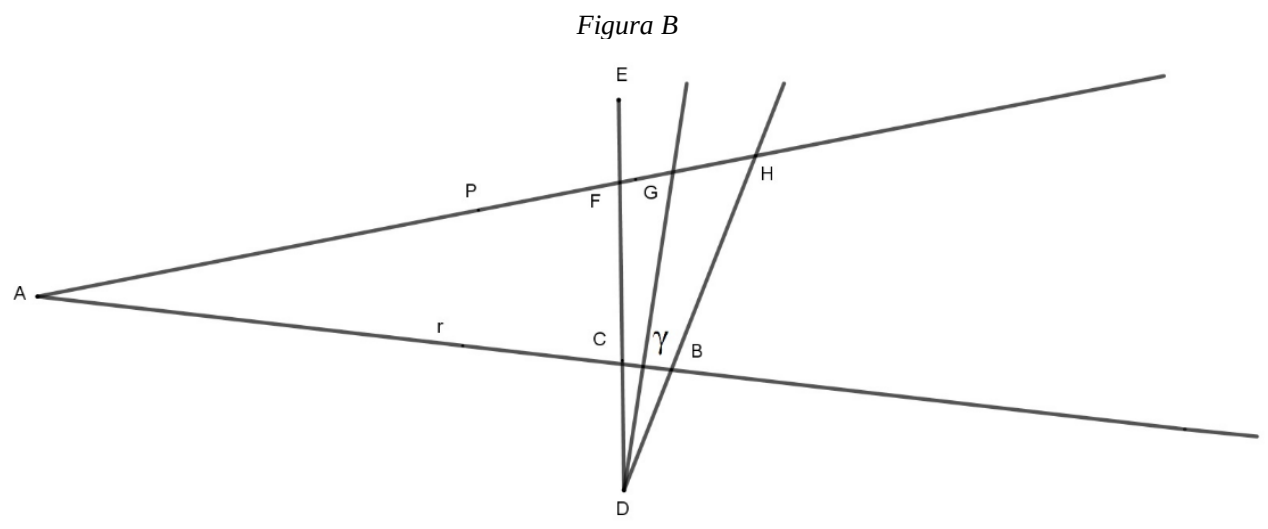

Figura C

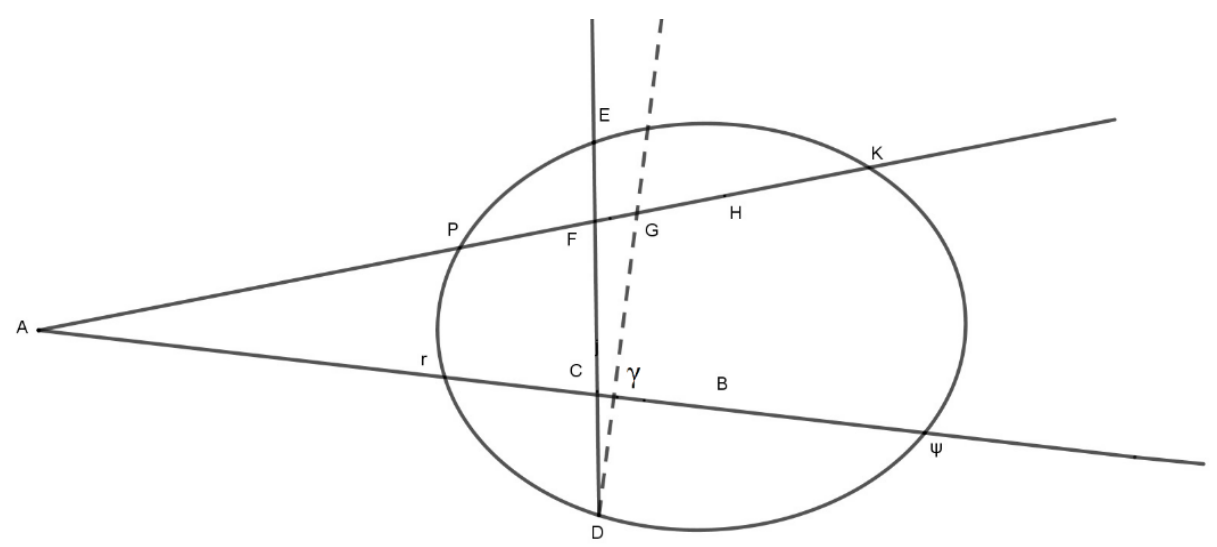

Figura D

RBHM, Vol. 21, n 42, pp. 180-205, 2021 
João F. N. B. Cortese

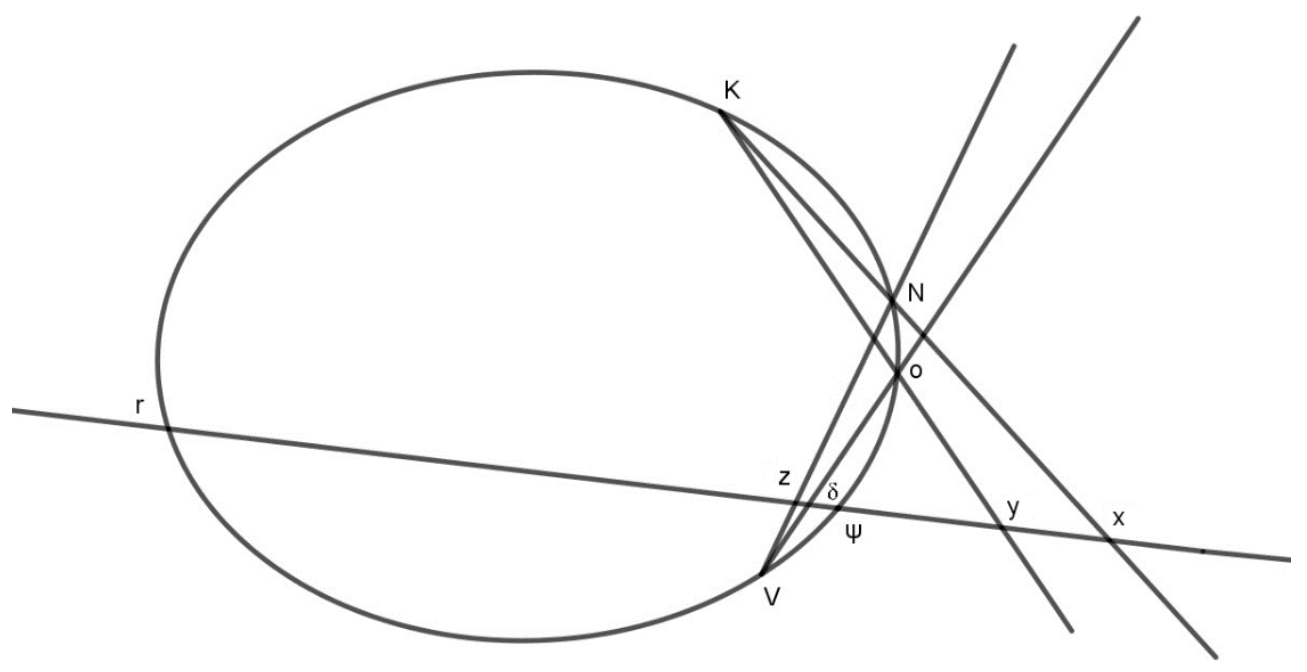

Figura E 


\section{Tradução}

\section{ENSAIO PARA AS CÔNICAS}

Por B. P.

\section{DEFINIÇÃO PRIMEIRA}

Quando várias linhas retas concorrem em um mesmo ponto, ou são todas paralelas entre elas, todas essas linhas são ditas de mesma ordem ou de mesma ordenança ${ }^{22}$, e a multiplicidade ${ }^{23}$ dessas linhas é dita ordem de linhas, ou ordenança de linhas.

\section{DEFINIÇÃO II}

Pelo termo de seção de cone, entendemos a circunferência do círculo, a elipse, a hipérbole, a parábola e o ângulo retilíneo, na medida em que um cone, cortado paralelamente à sua base, ou por seu vértice, ou em três outros sentidos que engendram a elipse, a hipérbole e a parábola, engendra na superfície cônica, ou a circunferência de um círculo, ou um ângulo, ou a elipse, ou a hipérbole, ou a parábola ${ }^{24}$.

\section{DEFINIÇÃO III}

Pelo termo de reta, tomado isoladamente, entendemos linha reta.

\section{LEMA I}

Se, no plano MSQ, do ponto $\mathrm{M}$ partem as duas retas MK, MV, e do ponto S partem as duas retas SK, SV, e sendo $\mathrm{K}$ a concorrência das retas MK, SK, e V a concorrência das retas $\mathrm{MV}, \mathrm{SV}$, e A a concorrência das retas $\mathrm{MK}, \mathrm{SV}$, e $\mu$ a concorrência das retas $\mathrm{MV}$, SK, e que por dois dos quatro pontos $\mathrm{A}, \mathrm{K}, \mu, \mathrm{V}$, que não estejam em uma mesma reta com os pontos $\mathrm{M}, \mathrm{S}$, como pelos pontos $\mathrm{K}, \mathrm{V}$, passe a circunferência de um

\footnotetext{
${ }^{22}$ Traduzimos ordonnance por “ordenança”. A primeira definição é tomada de Desargues (ver o texto do Brouillon project... em TATON, 1951, p. 100). Como indica J. Mesnard (em OC, II, p. 231), é Pascal que introduz o termo ordre, ao lado da ordonnance da qual falava Desargues. Hoje se usa o termo "feixe de retas".

${ }^{23}$ No francês, multitude. O sentido é do conjunto das diversas retas; preferimos manter um termo de mesma raiz.

${ }^{24}$ Esta concepção geral das cônicas também é devida a Desargues (ver Mesnard, em OC, II, p. 231).
} 
círculo, que corta as retas $\mathrm{MV}, \mathrm{MK}, \mathrm{SV}, \mathrm{SK}$, nos pontos $\mathrm{O}, \mathrm{P}, \mathrm{Q}, \mathrm{N}$; digo que as retas $\mathrm{MS}$, NO, PQ, são de mesma ordem ${ }^{25} 26$. [fig. I / fig. A]

\section{LEMA II}

Se pela mesma reta passam vários planos, sendo cortados por um outro plano, todas as linhas das seções desses planos são de mesma ordem com a reta pela qual passam os planos referidos.

Esses dois lemas sendo postos, e algumas consequências fáceis deles, demonstraremos que, as mesmas coisas do primeiro lema sendo postas, se pelos pontos $\mathrm{K}$, $\mathrm{V}$, passa uma seção de cone qualquer que corta as retas MK, MV, SK, SV, nos pontos $\mathrm{P}, \mathrm{O}$, $\mathrm{N}, \mathrm{Q}^{27}$, as retas $\mathrm{MS}, \mathrm{NO}, \mathrm{PQ}$, serão de mesma ordem. Isso será um terceiro lema ${ }^{28}$. [fig. I / fig. A]

Em seguida a esses três lemas, e a algumas consequências deles, apresentaremos Elementos cônicos completos, a saber, todas as propriedades dos diâmetros e lados retos ${ }^{29}$, das tangentes, etc., a restituição de quase todos os dados do cone ${ }^{30}$, a descrição das seções de cone por pontos ${ }^{31}$, etc.

\footnotetext{
${ }^{25}$ Segundo a primeira definição (retas de mesma ordem), isso significa que as retas MS, NO e PQ são concorrentes em um mesmo ponto, ou paralelas. Ou seja, a intersecção (dos prolongamentos) de NO e PQ se encontrará sobre (o prolongamento de) MS: os (prolongamentos dos) lados opostos do hexágono inscrito ONKPQV se intersectam sobre a mesma reta (cf. Mesnard, em OC, II, p. 231). Sem fazer menção a um hexágono, Pascal já apresenta aqui, para o caso do círculo, um resultado que pode ser considerado equivalente ao que será depois chamado de Teorema de Pascal ou Teorema do Hexágono (ver nossa seção 1.4). Vemos que o caso das retas paralelas é tratado aqui conjuntamente com o caso das retas concorrentes, por meio da definição de retas "de mesma ordem". Na Generatio conisectionum, Pascal tratará tal unificação por meio da noção de distância infinita: "Duas ou mais retas, qualquer que seja a sua posição, são sempre ditas concorrentes, seja a distância finita, se elas se cortam em um mesmo ponto, seja a distância infinita, se elas são paralelas” (OC, II, p. 1111). Neste sentido, pode-se entender que o ponto de intersecção das retas paralelas está no infinito (o que corresponde ao caso do hexágono regular no lema I do Ensaio para as cônicas).

${ }^{26}$ Diversas demonstrações foram propostas ao Teorema de Pascal. Houzel (2013) realiza uma derivação do Lema I a partir do Teorema de Menelau (ver abaixo, nota 38), da recíproca de um lema de Pappus (proposições 136 e 142 do livro VII da Coleção) e da propriedade de potência de um ponto em relação a uma circunferência.

${ }^{27}$ Mesnard omite aqui o ponto P. Entendemos que se trata de um lapso, pois o ponto P é mencionado no texto original.

${ }^{28} \mathrm{O}$ terceiro lema pode ser entendido como uma extensão do lema I, no caso em que se substitui o círculo PKVQ por uma cônica qualquer. Se tomarmos esta cônica como a projeção de um círculo, e levando em conta que a propriedade enunciada é invariante sob projeção, o lema III pode ser derivado do lema I (HOUZEL, 2013, pp. 9394).

29 “O lado reto, também chamado de parâmetro (termo que acabará por prevalecer), define-se assim no século XVII: sendo dados dois diâmetros conjugados de uma cônica, de comprimentos $2 a^{\prime}$ e $2 b^{\prime}$, o lado reto do primeiro desses diâmetros é o segmento de comprimento $2 \frac{b^{12}}{a^{1}}$ ”(Mesnard, OC, II, p. 232, nota; ver também TATON, 1955, p. 13).

30 “Ou seja, a determinação dos vértices dos cones passando por uma cônica definida por certos dados” (TATON, 1955, p. 13).
} 
Tendo feito isso, enunciamos as propriedades que tratamos aí de maneira mais universal do que é ordinário. Por exemplo, esta: se no plano MSQ, na seção de cone PKV, são traçadas as retas $\mathrm{AK}, \mathrm{AV}$, que atingem a seção nos pontos $\mathrm{PK}, \mathrm{QV}^{32}$; e que de dois desses quatro pontos que não estão em mesma reta com o ponto $\mathrm{A}$, como pelos pontos $\mathrm{K}$, $\mathrm{V}$, e por dois pontos $\mathrm{N}, \mathrm{O}$, tomados no contorno da seção, sejam traçadas quatro retas $\mathrm{KN}$, $\mathrm{KO}, \mathrm{VN}, \mathrm{VO}$, cortando as retas $\mathrm{AV}, \mathrm{AP}$, nos pontos $\mathrm{L}, \mathrm{M}, \mathrm{T}, \mathrm{S}^{33}$ : digo que a razão composta $^{34}$ das razões da reta $\mathrm{PM}$ à reta $\mathrm{MA}$, e da reta $\mathrm{AS}$ à reta $\mathrm{SQ}$, é a mesma que a composta das razões da reta $\mathrm{PL}$ à reta $\mathrm{LA}$, e da reta $\mathrm{AT}$ à reta $\mathrm{TQ}^{35}$. [fig. I / fig. B]

Demonstraremos também que, se há três retas DE, DG, DH, que as retas AP, $\mathrm{Ar}^{36}$, cortam nos pontos F, G, H, C, ү, B, e que na reta DC seja determinado o ponto E; a razão composta das razões do retângulo ${ }^{37} \mathrm{EF}$ em FG ao retângulo de EC em Cү, e da reta Ay à reta AG, é a mesma que a composta das razões do retângulo EF em FH ao retângulo EC em $\mathrm{CB}$, e da reta $\mathrm{AB}$ à reta $\mathrm{AH}$. E é também a mesma que a razão do retângulo das retas $\mathrm{FE}$, FD, ao retângulo das retas $\mathrm{CE}, \mathrm{CD}^{38}$. Portanto, se pelos pontos E, D, passa uma seção de

31 “Ou seja, a construção por pontos de cônicas definidas por certos dados (por exemplo $n$ pontos e (5- $n$ ) tangentes)” (TATON, 1955, p. 13).

${ }^{32}$ Entende-se que se trata dos quatro pontos $\mathrm{P}, \mathrm{K}, \mathrm{Q}, \mathrm{V}$, colineares dois a dois.

33 Taton (1955, p. 13) corrige para “S, T, L, M", a fim de preservar uma correspondência entre a ordem dos pontos e a ordem das retas. Ele indica, entretanto, que a figura de Pascal está correta.

${ }^{34}$ Podemos entender, anacronicamente, a "razão composta” como o produto de duas razões.

${ }^{35}$ Primeiro teorema, cujo resultado pode ser assim expresso:

$$
\frac{P M}{M A} \times \frac{A S}{S Q}=\frac{P L}{L A} \times \frac{A T}{T Q}
$$

O que pode ser visto como equivalente a afirmar a igualdade das razões anarmônicas dos pontos (PAML) e (AQTS). Segundo Taton (1955, p. 14), tal teorema pode ser obtido por extensão projetiva do caso do círculo (Lema 1), ou partindo do Teorema de Pascal, ou ainda do Teorema de Desargues. Houzel (2013) propõe uma demonstração a partir de lemas de Pappus (proposições 129, 136 e 142 do livro VII da Coleção).

${ }^{36}$ Seguimos a grafia de Mesnard, sempre grafando $r$ em minúscula. Mesnard nota que na figura I a referência ao ponto $r$ é feita pela letra minúscula, enquanto no corpo do texto ele era retomado na publicação original tanto em maíscula quanto em minúscula. Mesnard opta pela uniformização em minúscula pelo fato de que os seis pontos da figura que estariam "em involução”, segundo a terminologia de Desargues, são intencionalmente distinguidos pelas letras minúsculas: $\mathrm{r}, \mathrm{z}, \delta, \psi, \mathrm{y}$, x. Lembremos que, para Desargues, a disposição de três pares de pontos B, H; C, G; D, F; todos em uma mesma linha reta, é chamada de "involução" no caso em que (em formulação anacrônica):

$$
\frac{G D \times G F}{C D \times C F}=\frac{G B \times G H}{C B \times C H}
$$

(cf. FIELD e GRAY, 1987, p. 48; para a formulação original de Desargues, ver FIELD e GRAY, 1987, p. 77 e TATON, 1951, p. 110).

37 Podemos entender, anacronicamente, o “retângulo" entre duas retas como o seu produto - terminologia evidentemente ligada à geometria, se consideramos um retângulo que tem como comprimento uma das duas retas consideradas e como largura a outra.

${ }^{38}$ Primeira parte do segundo teorema, cujo resultado pode ser assim expresso:

$$
\frac{E F \times F G}{E C \times C \gamma} \times \frac{A \gamma}{A G}=\frac{E F \times F H}{E C \times C B} \times \frac{A B}{A H}=\frac{E F \times F D}{E C \times C D}
$$


cone que corta as retas $\mathrm{AH}, \mathrm{AB}$, pelos pontos $\mathrm{P}, \mathrm{K}, \mathrm{r}, \psi$, a razão composta das razões do retângulo das retas $\mathrm{EF}, \mathrm{FG}$, ao retângulo das retas $\mathrm{EC}, \mathrm{C} \gamma$, e da reta $\gamma \mathrm{A}$ à reta $\mathrm{AG}$, será a mesma que a composta das razões do retângulo das retas FK, FP ao retângulo das retas Cr, $\mathrm{C} \psi$, e do retângulo das retas $\mathrm{Ar}, \mathrm{A} \psi$, ao retângulo das retas $\mathrm{AK}, \mathrm{AP}^{39}$. [fig. I / fig. C, D]

Demonstraremos também que se quatro retas AC, AF, EH, EL, se entrecortam nos pontos $\mathrm{N}, \mathrm{P}, \mathrm{M}, \mathrm{O}$, e que uma seção de cone corta as referidas retas nos pontos $\mathrm{C}, \mathrm{B}, \mathrm{F}, \mathrm{D}$, H, G, L, K, a razão composta das razões do retângulo de MC em MB, ao retângulo das retas $\mathrm{PF}, \mathrm{PD}$, e do retângulo das retas $\mathrm{AD}, \mathrm{AF}$, ao retângulo das retas $\mathrm{AB}, \mathrm{AC}$, é a mesma que a razão composta das razões do retângulo das retas $\mathrm{ML}, \mathrm{MK}$, ao retângulo das retas PH, PG, e do retângulo das retas EH, EG, ao retângulo das retas EK, EL ${ }^{40}$. [fig. III]

Demonstraremos também esta propriedade, cujo primeiro inventor é o Sr. Desargues, de Lyon, um dos grandes espíritos deste tempo, e dos mais versados em matemáticas, e entre outras nas cônicas, cujos escritos sobre essa matéria, embora em pequeno número, deram um amplo testemunho àqueles que quiseram dela receber a compreensão; e quero de fato admitir que devo o pouco que encontrei sobre essa matéria a seus escritos, e que tentei imitar, tanto quanto me foi possível, seu método sobre esse tema, que ele tratou sem se servir do triângulo pelo eixo ${ }^{41}$. E, tratando em sua generalidade todas

É importante retomar aqui o Teorema de Menelau, atribuído por Desargues a Ptolomeu (HOUZEL, 2013, p. 92). Tal teorema aparecia no terceiro livro das Esféricas de Menelau, um trabalho editado pela primeira vez por Maurolico em 1588 na sua Cosmographia (DEL CENTINA, 2020): ele enuncia que, se AB e AG são duas retas e $\mathrm{BE}, \mathrm{GD}$ são outras duas retas, cuja intersecção de uma à outra se dá em $\mathrm{F}$, e cujas intersecções às retas $\mathrm{AB}$, AG se dão respectivamente em D e E, então

$$
\frac{G E}{E A}=\frac{G F}{D F} \times \frac{B D}{B A}
$$

Quanto ao resultado de Pascal, "a igualdade entre o primeiro e o terceiro membro da equação resulta da aplicação do teorema de Ptolomeu-Menelau ao triângulo AFC, cortado pela secante GүD; a igualdade entre o segundo e o terceiro membros, da aplicação do mesmo teorema ao triângulo AFC, cortado pela secante HBD” (TATON, 1955, p. 15; ver também DEL CENTINA, 2020). Taton nota, finalmente, que Desargues emprega frequentemente este teorema no seu Brouillon project.

${ }^{39}$ Segunda parte do segundo teorema, cujo resultado pode ser assim expresso:

$$
\frac{E F \times F G}{E C \times C \gamma} \times \frac{A \gamma}{A G}=\frac{F K \times F P}{C r \times C \psi} \times \frac{A r \times A \psi}{A K \times A P}
$$

A comparação desta igualdade com a precedente (ver acima, nota 38) permite eliminar a intervenção da secante DyG e conduz assim a um caso particular do Teorema de Carnot (CARNOT, 1806, n. 378; cf. TATON, 1955, p. 15 e DEL CENTINA, 2020).

${ }^{40}$ Terceiro teorema, cujo resultado pode ser assim expresso:

$$
\frac{M C \times M B}{P F \times P D} \times \frac{A D \times A F}{A B \times A C}=\frac{M L \times M K}{P H \times P G} \times \frac{E H \times E G}{E K \times E L}
$$

Este terceiro teorema é também um caso particular do Teorema de Carnot (CARNOT, 1806, n. 379; cf. TATON, 1955).

${ }^{41}$ Segundo Taton (1955, p. 205), “o método dito ‘do triângulo pelo eixo’ era o fundamento do estudo das cônicas por Apolônio” - Taton cita (CHASLES, 1875, pp. 18-19) - "e ele havia sido adotado até então por todos os 
as seções de cone, a propriedade maravilhosa da qual é questão é esta. Se no plano MSQ há uma seção de cone PQV, no contorno da qual, tendo tomado os quatro pontos $\mathrm{K}, \mathrm{N}, \mathrm{O}, \mathrm{V}$, são traçadas as retas $\mathrm{KN}, \mathrm{KO}, \mathrm{VN}$, $\mathrm{VO}$, de modo que por um mesmo dos quatro pontos passem apenas duas retas, e que uma outra reta corte tanto no contorno da seção, nos pontos $\mathrm{r}, \psi$, quanto as retas $\mathrm{KN}, \mathrm{KO}, \mathrm{VN}, \mathrm{VO}$, nos pontos $\mathrm{x}, \mathrm{y}, \mathrm{z}, \delta$ : digo que, como o retângulo das retas zr, z $\psi$, está para o retângulo das retas yr, y $\psi$, assim o retângulo das retas $\delta r, \delta \psi$, está para o retângulo das retas $\mathrm{xr}, \mathrm{x} \psi^{42}$. [fig. I / fig. E]

Demonstraremos também que, se no plano da hipérbole, ou da elipse, ou do círculo, $\mathrm{AGE}$, cujo centro é $\mathrm{C}$, traça-se a reta $\mathrm{AB}$, tangente à seção no ponto $\mathrm{A}$, e, tendo traçado o diâmetro $\mathrm{CA}$, toma-se a reta $\mathrm{AB}$, cujo quadrado é igual ao quarto do retângulo da figura $^{43}$, e que se trace $\mathrm{CB}$, então, para qualquer reta que se trace, como DE, paralela à reta $A B$, cortando a seção em E, e as retas AC, CB, nos pontos D, F, se a seção AGE for uma elipse ou um círculo, a soma dos quadrados das retas DE, DF, será igual ao quadrado da reta $\mathrm{AB}$; e na hipérbole, a diferença dos mesmos quadrados das retas $\mathrm{DE}$, DF, será igual ao quadrado da reta $\mathrm{AB}^{44}$. [fig. II]

Deduziremos assim alguns problemas, por exemplo: De um ponto dado, traçar uma reta tangente a uma seção de cone dada.

Encontrar dois diâmetros conjugados em ângulo dado.

Encontrar dois diâmetros em ângulo dado e em razão dada.

Temos vários outros problemas e teoremas, e várias consequências dos precedentes; mas a desconfiança que tenho de minha pouca experiência e capacidade não me permite apresentar mais deles antes de ter passado pelo exame das hábeis pessoas que poderão nos tornar agradecidos a tomar a pena; após o que, se se julgar que a coisa merece ser continuada, tentaremos levá-la até onde Deus nos dará a força de conduzi-la.

PARIS, M. DC. XL.

autores do século XVII, à exceção de Desargues”.

${ }^{42}$ Quarto teorema, correspondente ao célebre teorema de Desargues sobre a "involução" (ver acima, nota 36), termo que Pascal não retoma aqui. O enunciado de Pascal está incorreto, pois, como mostrou TATON (1955, p. 16), ao invés de

$$
\frac{P M}{M A} \times \frac{A S}{S Q}=\frac{P L}{L A} \times \frac{A T}{T Q}
$$

deveria-se escrever

$$
\frac{G D \times G F}{C D \times C F}=\frac{G B \times G H}{C B \times C H}
$$

43 “O 'retângulo da figura' é para Pascal o produto do diâmetro diâmetro considerado (aqui, o dobro de CA) por seu parâmetro ou lado reto (no sentido da época)” (TATON, 1955, p. 17; ver acima, nota 29).

44 Quinto teorema, que permite encontrar a equação de uma seção cônica central, tendo como eixos de coordenadas dois diâmetros conjugados. Para detalhes, ver (TATON, 1955, p. 17; HOUZEL, 2013, pp. 100-101). Hara (1981, p. 25) sugere uma demonstração a partir do segundo teorema. Houzel (2013, p. 100) indica que, diferentemente das propriedades anteriores, que eram projetivas, esta é uma propriedade afim.

RBHM, Vol. 21, n 42, pp. 180-205, 2021 


\section{Texto original $^{45}$}

\section{ESSAI POUR LES CONIQUES}

Par B. P.

\section{DÉFINITION PREMIÈRE}

Quand plusieurs lignes droites concourent à même point, ou sont toutes parallèles ${ }^{46}$ entre elles, toutes ces lignes sont dites de même ordre ou de même ordonnance, et la multitude de ces lignes est dite ordre de lignes, ou ordonnance de lignes.

\section{DÉFINITION II}

Par le mot de section de cône, nous entendons la circonférence du cercle, l'ellipse, l'hyperbole, la parabole et l'angle rectiligne, d'autant qu'un cône coupé parallèlement à sa base, ou par son sommet, ou des trois autres sens qui engendrent l'ellipse, l'hyperbole et la parabole, engendre dans la superficie conique, ou la circonférence d'un cercle, ou un angle, ou l'ellipse, ou l'hyperbole, ou la parabole.

\section{DÉFINITION III}

Par le mot de droite mis seul, nous entendons ligne droite.

\section{LEMME I}

Si dans le plan MSQ du point M partent les deux droites MK, MV, et du point S partent les deux droites SK, SV, et que K soit le concours des droites MK, SK, et V le concours des droites $\mathrm{MV}, \mathrm{SV}$, et $\mathrm{A}$ le concours des droites $\mathrm{MK}, \mathrm{SV}^{47}$, et $\mu$ le concours des droites MV, SK, et que par deux des quatre points $\mathrm{A}, \mathrm{K}, \mu, \mathrm{V}$, qui ne soient point en même droite avec les points $\mathrm{M}$, S, comme par les points $\mathrm{K}, \mathrm{V}$, passe la circonférence d'un cercle, coupante les droites $\mathrm{MV}, \mathrm{MK}^{48}$, SV, SK, ès points $\mathrm{O}, \mathrm{P}, \mathrm{Q}, \mathrm{N}$, je dis que les droites $\mathrm{MS}$, NO, PQ, sont de même ordre. [En marge: fig. I / fig. A]

\section{LEMME II}

${ }^{45}$ Edição de J. Mesnard em PASCAL 1964-1992, vol. II, pp. 228-235.

${ }^{46}$ Nota de Mesnard: no original, paralleles.

${ }^{47}$ Nota de Mesnard: no original, "MA, SA".

${ }^{48}$ Nota de Mesnard: no original, "MP”. 
Si par la même droite passent plusieurs plans, qui soient coupés par un autre plan, toutes les lignes des sections de ces plans sont de même ordre avec la droite par laquelle passent lesdits plans.

Ces deux lemmes posés, et quelques faciles conséquences d'iceux, nous démontrerons que, les mêmes choses étant posées qu'au premier lemme, si par les points K, $\mathrm{V}$, passe une quelconque section de cône qui coupe les droites MK, MV, SK, SV ès points $\mathrm{O}, \mathrm{N}, \mathrm{Q}^{49}$, les droites MS, NO, PQ, seront de même ordre. Cela sera un troisième lemme. [En marge: fig. I / fig. A]

En suite de ces trois lemmes et de quelques conséquences d'iceux, nous donnerons des Éléments coniques complets, à savoir toutes les propriétés des diamètres et côtés droits, des tangentes, etc., la restitution du cône presque sur toutes les données, la description des sections de cône par points, etc.

Quoi faisant, nous énonçons les propriétés que nous en touchons d'une manière plus universelle qu'à l'ordinaire. Par exemple, celle-ci: si dans le plan MSQ, dans la section de cône PKV, sont menées les droites AK, AV, atteignantes la section aux points PK, QV ; et que de deux de ces quatre points qui ne sont point en même droite avec le point $A$, comme par les points $\mathrm{K}, \mathrm{V}$, et par deux points $\mathrm{N}$, O, pris dans le bord de la section, soient menées quatre droites $\mathrm{KN}, \mathrm{KO}, \mathrm{VN}$, $\mathrm{VO}$, coupantes les droites $\mathrm{AV}$, AP aux points $\mathrm{L}, \mathrm{M}, \mathrm{T}$, $\mathrm{S}$ : je dis que la raison composée des raisons de la droite PM à la droite MA, et de la droite AS à la droite SQ, est la même que la composée des raisons de la droite PL à la droite LA, et de la droite AT à la droite TQ. [En marge: fig. I / fig. B]

Nous démontrerons aussi que s'il y a trois droites DE, DG, DH, que les droites AP, $\mathrm{Ar}^{50}$, coupent aux points $\mathrm{F}, \mathrm{G}, \mathrm{H}, \mathrm{C}, \gamma, \mathrm{B}$, et que dans la droite $\mathrm{DC}$ soit déterminé le point $\mathrm{E}$, la raison composée des raisons du rectangle EF en FG au rectangle de EC en $\mathrm{C} \gamma$, et de la droite $\mathrm{Ay}$ à la droite $\mathrm{AG}$, est la même que la composée des raisons du rectangle EF en FH au rectangle $\mathrm{EC}$ en $\mathrm{CB}$, et de la droite $\mathrm{AB}$ à la droite $\mathrm{AH}$. Et est aussi la même que la raison du rectangle des droites FE, FD, au rectangle des droites CE, CD. Partant, si par les points $E, D$, passe une section de cône qui coupe les droites $A H, A B$, ès points $P, K, r, \psi$, la raison composée des raisons du rectangle des droites $\mathrm{EF}, \mathrm{FG}^{51}$, au rectangle des droites $\mathrm{EC}, \mathrm{C} \gamma$, et de la droite $\gamma \mathrm{A}$ à la droite AG, sera la même que la composée des raisons du rectangle des droites FK, FP, au rectangle des droites $\mathrm{Cr}$, $\mathrm{C} \psi$, et du rectangle des droites $\mathrm{Ar}, \mathrm{A} \psi$, au rectangle des droites AK, AP. [En marge: fig. I / fig. C, D]

\footnotetext{
${ }^{49}$ Mesnard omite aqui o ponto P. Entendemos que se trata de um lapso, pois o ponto P é mencionado no texto original.

${ }^{50}$ Nota de Mesnard: no original, "R”. Idem para as ocorrências seguintes.

${ }^{51}$ Nota de Mesnard: no original, "FC”.
}

RBHM, Vol. 21, n 42, pp. 180-205, 2021 
Nous démontrerons aussi que si quatre droites AC, AF, EH, EL, s'entrecoupent ès points $\mathrm{N}, \mathrm{P}, \mathrm{M}, \mathrm{O}$, et qu'une section de cône coupe lesdites droites ès points $\mathrm{C}, \mathrm{B}, \mathrm{F}^{52}, \mathrm{D}, \mathrm{H}$, $\mathrm{G}, \mathrm{L}, \mathrm{K}$, la raison composée des raisons du rectangle de $\mathrm{MC}$ en $\mathrm{MB}$, au rectangle des droites $\mathrm{PF}, \mathrm{PD}$, et du rectangle des droites $\mathrm{AD}, \mathrm{AF}$, au rectangle des droites $\mathrm{AB}, \mathrm{AC}$, est la même que la raison composée des raisons du rectangle des droites ML, MK, au rectangle des droites PH, PG, et du rectangle des droites EH, EG, au rectangle des droites EK, EL. [En marge: fig. III]

Nous démontrerons aussi cette propriété, dont le premier inventeur est M. Desargues, Lyonnais, un des grands esprits de ce temps et des plus versés aux mathématiques, et entre autres aux coniques, dont les écrits sur cette matière, quoique en petit nombre, en ont donné un ample témoignage à ceux qui en auront voulu recevoir l'intelligence ; et veux bien avouer que je dois le peu que j'ai trouvé sur cette matière à ses écrits, et que j'ai tâché d'imiter, autant qu'il m’a été possible, sa méthode sur ce sujet, qu'il a traité sans se servir du triangle par l'axe. Et, traitant généralement de toutes les sections de cône, la propriété merveilleuse dont est question est telle. Si dans le plan MSQ y a une section de cône PQV, dans le bord de laquelle ayant pris les quatre points $\mathrm{K}, \mathrm{N}, \mathrm{O}$, V, sont menées les droites $\mathrm{KN}, \mathrm{KO}, \mathrm{VN}$, $\mathrm{VO}$, de sorte que par un même des quatre points ne passent que deux droites, et qu'une autre droite coupe tant le bord ${ }^{53}$ de la section aux points $\mathrm{r}, \psi$, que les droites $\mathrm{KN}, \mathrm{KO}, \mathrm{VN}, \mathrm{VO}$, ès points $\mathrm{x}, \mathrm{y}, \mathrm{z}^{54}, \delta:$ je dis que, comme le rectangle des droites zr, z $\psi$ est au rectangle des droites $\mathrm{yr}^{55}, \gamma \psi$, ainsi le rectangle des droites $\delta r, \delta \psi$ est au rectangle des ${ }^{56}$ droites $\mathrm{xr}, \mathrm{x} \psi$. [En marge: fig. I / fig. E]

Nous démontrerons aussi que, si dans le plan de l'hyperbole ou de l'ellipse, ou du cercle AGE, dont le centre est $C$, on mène la droite $A B$, touchante au point $A$ la section, et qu'ayant mené le diamètre $C A$, on prenne la droite $A B$ dont le carré soit égal au quart du rectangle de la figure, et qu'on mène $\mathrm{CB}$, alors, quelque droite qu'on mène, comme $\mathrm{DE}$, parallèle à la droite $\mathrm{AB}$, coupante la section en $\mathrm{E}$, et les droites $\mathrm{AC}, \mathrm{CB}$, ès points $\mathrm{D}, \mathrm{F}$, si la section AGE est une ellipse ou un cercle, la somme des carrés des droites DE, DF, sera égale au carré de la droite $A B$; et dans l'hyperbole, la différence des mêmes carrés des droites DE, DF, sera égale au carré de la droite AB. [En marge: fig. II]

Nous déduirons aussi quelques problèmes, par exemple : D’un point donné mener une droite touchante une section de cône donnée.

Trouver deux diamètres conjugués en angle donné.

Trouver deux diamètres en angle donné et en raison donnée.

\footnotetext{
${ }^{52}$ Nota de Mesnard: no original, "E”.

${ }^{53}$ Nota de Mesnard: no original, "l'abord".

${ }^{54}$ Nota de Mesnard: no original, “Z” em maiúscula. Idem para a sequência.

${ }^{55} \mathrm{O}$ texto de Mesnard apresenta um equívoco aqui, escrevendo yr; corrigimos para yr, de acordo com a própria imagem da publicação orginal e com a interpretação proposta por DEL CENTINA, 2000. Talvez a edição de Mesnard seja influenciada pelo fato de que em TATON (1955) a escrita não é clara, as letras y do trecho "yr, y $\psi$ " sendo grafadas uma em negrito e a outra não; além disso, os sinais y e $\gamma$ são evidentemente parecidos, e $\gamma$ havia sido usado na proposição 2 .

${ }^{56} \mathrm{O}$ termo des foi omitido no original, e é acrescentado por Mesnard.
} 
Nous avons plusieurs autres problèmes et théorèmes, et plusieurs conséquences des précédents ; mais la défiance que j’ai de mon peu d'expérience et de capacité ne me permet pas d'en avancer davantage avant qu'il ait passé à l'examen des habiles gens qui voudront nous obliger d'en prendre la peine : après quoi, si l'on juge que la chose mérite d'être continuée, nous essaierons de la pousser jusques où Dieu nous donnera la force de la conduire.

À PARIS, M. DC. XL. 